\title{
Targeting BMI1 and MCL1 for Lung
}

\section{Adenocarcinoma Treatment}

Erh-Hsuan Lin, Chiung-Fang Hsu, Yi-Hua J an, Tsung-Hsien Lin, Hsiang-Yi Ch ang, Chun-Ming Cheng, Hui-J an Hsu, Wei-Wei Chen, Bo-Hung Chen, Hsin

g-Fang Tsai, J ung-J ung Li, Chi-Ying Huang, Shih-Hsien Chuang, J ia-Ming

Chang, Mic hael Hsiao, Cheng-Wen Wu

This manuscript has been withdrawn by the author as their new findings will restructure the article. The author does not wish this work to be cited currently as reference for the project. If you have any questions, please contact the corresponding author. 